\title{
RELAÇÃO ENTRE TEORIA, PESQUISA E PRÁTICA
}

Mercedes Trentini*

TRENTINI, M. Relação entre teoria, pesquisa e prática. Rev. Esc. Enf. USP, Sãoo Paulo, 21 (2):135-143, ago. 1987.

Este trabalho tem o objetivo de descrever o processa de interrelação da teoria, pesquisa e prática na enfermagem. Para isso, inicialmente, define-se o termo "teoria" $e$ os componentes principais de uma teoria. Os conceitos de pesquisa e prática são discutidos em termos de sua potencialidade de gerar, testar $e$ redefinir a teoria. Finalmente, os conceitos teoria, pesquisa e prática são apresentados formando uma espiral triplice onde interagem para aperfeiçoamento mutuo. enfermagem.

UNITERMOS: Teoria de enfermagem. Pesquisa em enfermagem. Prática de

\section{INTRODUÇÃO}

O homem adquire conhecimento através de diversas maneiras as quais incluem: tradição, autoridade, experiência da vida diária ou pela maneira mais comum, no meio profissional, que é através do conhecimento sistematizado que inclui o desenvolvimento de teorias e pesquisas.

Durante as duas últimas décadas, profissionais da área de enfermagem têm empregado enorme esforço para desenvolver um corpo de conhecimentos específicos para orientar a prática de enfermagem. Esse esforço resultou no desenvolvimento de várias teorias e de inúmeros trabalhos de pesquisa, apesar disso, a prática da enfermagem ainda continua desvinculada das bases teóricas próprias da enfermagem. Essa lacuna entre teoria e prática na enfermagem será preenchida à medida que houver uma estreita relação entre teoria, pesquisa e prática. $O$ objetivo deste artigo, portanto, consiste em apresentar o processo da relação entre teoria, pesquisa e prática na enfermagem. A fim de facilitar a compreensão do assunto, é oportuno discutir antes, o significado do termo "teoria".

\section{Teoria}

Em situações diárias, o termo teoria é, geralmente, usado para designar uma crença ou uma idéia. Teoria é também um termo popularmente usado para denominar o oposto da prática. Por exemplo, um currículo de graduação em enfermagem contém disciplinas ditas de carga teórica e prática. Neste caso, o desenvolvimento da carga teórica é, geralmente, chamado teoria. O termo teoria tem também uma outra conotação a qual designa a teoria como sendo um componente do conhecimento científico. Dentro desta concepção, teoria é caracterizada pela sua construção, a qual requer o uso de um método próprio.

* Enfermeira. Professora do Departamento de Enfermagem/Curso de Mestrado - UFSC. Doutora em Enfermagem pela Universidade do Alabama em Birminghann, USA. 
Inúmeras definições do termo teoria são encontradas na literatura. Para CHINN \& JACOBS ${ }^{5}$ teoria é uma abstração sistematizada da realidade. Teoria tem sido também definida como sendo um conjunto de representações simbólicas de: a) relações observadas e/ou inferidas entre eventos, b) estruturas pressupostas que fundamentam essas relações e c) descriçōes básicas que têm a finalidade de explicar eventos na ausência de manifestações empíricas das relações entre eventos ${ }^{12}$. Segundo KERLINGER 11 teoria "é um conjunto de conceitos, definições, e proposições que apresentam uma visão sistemática de fenômenos através da especificação das relações entre os conceitos..." (p. 9). O objetivo da teoria, segundo KERLINGER ${ }^{11}$, é descrever, explicar, predizer e controlar fenômenos. Entretanto, nem todas as teorias têm estas quatro funçōes. Existem teorias, por exemplo, que apenas descrevem; outras descrevem e explicam um fenômeno e outras, além destas funções, predizem e/ou controlam o fenômeno. Para melhor compreensão do termo teoria, torna-se necessário definir cada elemento que compõe a teoria, o que será apresentado a seguir.

\subsection{Conceitos}

Conceitos são abstraçōes ou imagens mentais de objetos, propriedades, ou eventos que simbolizam a realidade ${ }^{10}$. Quando alguém pensa num objeto, como por exemplo uma seringa, imediatamente se forma uma imagem mental de um objeto constituido por duas partes, com formas cilindricas que se acoplam uma na outra. Essa imagem mental não é a seringa; ela simboliza a seringa. A seringa (objeto) é a realidade, e a imagem mental é uma abstração. Em outras palavras, a abstração ou a imagem mental da seringa é um conceito e o objeto (seringa) ẻ a realidade. Portanto, conceitos são representações da realidade. No exemplo acima, ocorreu uma formação mental da imagem do objeto (seringa), ou seja, formou-se a consciência de um conceito. Um conceito pode ser percebido por duas ou mais pessoas e cada pessoa pode ter uma percepção diferente do mesmo conceito. Isto quer dizer que um conceito pode ter diferentes significados. Por exemplo, para uma enfermeira, o conceito "seringa" pode significar um instrumento usado para aliviar ou curar uma dor. Para o paciente, por outro lado, a seringa pode significar um instrumento de tortura ou causador de dor. Observa-se que o mesmo conceito pode ter significados opostos, dependendo da percepção de cada pessoa.

Como vimos, conceitos são imagens mentais; portanto, todos os conceitos são abstratos. No entanto, há conceitos com diferentes niveis de abstração. CHINN \& JACOBS ${ }^{5}$ apresentam três categorias de conceitos, segundo seus niveis de abstração: 1) conceitos que podem ser diretamente observados, isto é, podem ser facilmente vistos, ouvidos ou percebidos. Nessa classificação podemos citar como exemplos, os conceitos de "seringa", "som" e "temperatura"; 2) conceitos que só podem ser observados indiretamente, através de sinais ou instrumentos. Por exemplo, o nível do potássio no sangue é um exemplo de conceito observado através de instrumentos; 3 ) conceitos que não podem ser claramente observados direta nem indiretamente, mas podem ser inferidos a partir de conceitos 
observáveis, como por exemplo, sentimento, são classificados dentro do mais auto nivel de abstração e são chamados construtos ${ }^{10}$.

Os construtos são conceitos formados por outros conceitos; por isso, são chamados construtos, significando "construídos por" ${ }^{10}$. Exemplo desse tipo de conceito é o termo "cultura". O conceito cultura inclui costumes, hábitos, crenças, normas, relacionamento. Como vemos, o conceito cultura abrange vários outros conceitos; em outras palavras, este conceito é construído por outros conceitos que neste caso são os subconceitos de cultura. Para definir cultura, há necessidade, antes, de definir os seus subconceitos. Portanto, cultura é um conceito altamente abstrato. Assim sendo, o construto "cultura" não pode ser observado direta ou indiretamente, mas pode-se fazer uma inferência a partir de observações dos seus subconceitos (hábitos, costumes, etc.). Como vimos acima, conceitos são abstrações, no entanto, REYNOLDS 18 classificou estas abstrações em dois sub-grupos, chamando de concretos os conceitos que dependem de tempo e espaço, e de abstratos os que independem destas condições. Por exemplo, segundo REYNOLDS ${ }^{18}$ o conceito "enfermagem" é abstrato e "enfermagem no Brasil" é concreto, porque depende de um espaço (Brasil). "Enfermagem na década de 70" é também um conceito concreto, porque depende de um tempo histórico.

Os conceitos são os elementos fundamentais de uma teoria. HARDY ${ }^{9}$ afirma que os conceitos são os blocos de construção de uma teoria. Ao tomarmos por base a classificação de conceitos segundoREYNOLDS ${ }^{18}$, pode-se afirmar que os conceitos usados na construção de teorias são os conceitos abstratos e que a abrangência de uma teoria depende da abstração dos seus conceitos. Por exemplo, uma teoria que incorpora o conceito "enfermagem" descreve e/ou explica a enfermagem independente de espaço ou tempo. Por outro lado, se uma outra teoria incluísse o conceito "enfermagem no Brasil", essa teoria seria limitada a descrever ou explicar somente a enfermagem no Brasil.

$E$ importante mencionar que um determinado conceito pode fazer parte de diferentes teorias. Nesse caso, o que difere é o significado atribuído ao conceito (usado em diferentes teorias) com outros conceitos de cada teoria. Os teoristas, ao elaborarem uma teoria, devem transmitir o significado de cada conceito, e isto é feito através das definições dos conceitos.

\subsection{Proposições}

O teorista, ao descrever as relações entre conceitos, está estabelecendo proposições. Um exemplo de proposição na teoria de enfermagem de Orem, referente ao conceito de auto-cuidado, é o seguinte: "As habilidades dos indivíduos de auto cuidar-se ou de depender do cuidado de outros são condicionadas pela idade, estado de desenvolvimento, experiência de vida, orientação sócio-cultural, saúde e recursos disponiveis" 16. Como vemos, nesta proposição o conceito de auto-cuidado está relacionado com os conceitos de idade, estado de desenvolvimento, experiência de vida, orientação sócio-cultural, saúde e recursos disponíveis. 


\subsection{Objetivos da teoria}

Segundo KERLINGER ${ }^{11}$, os objetivos da teoria consistem em descrever, explicar, predizer e controlar fenômenos. Uma teoria descreve um fenômeno, quando ela diz em que consiste o fenômeno. Quando uma teoria delineia o "porquê" da ocorrência do fenômeno e porque ocorre com certa regularidade, esta teoria explica o fenômeno ${ }^{11}$. A função preditiva de uma teoria é a potencialidade que a teoria tem de prever as condições sob as quais o fenômeno ocorre ${ }^{5}$. Uma teoria controla o fenômeno quando a teoria oferece condições para manipular o fenômeno a fim de evitar ou reduzir uma situação indesejada ${ }^{22}$.

\section{Niveis de Teoria}

Como foi descrito anteriormente, neste artigo, há diferentes niveis de abstrações de conceitos e, da mesma forma, existem diferentes níveis de teorias. WALKER \& AVANT ${ }^{21}$ descrevem quatro diferentes niveis de teorias. O primeiro é a Meta-teoria que trata de assunto filosóficos e metodológicos. As teorias de enfermagem a este nível, consistem em discussões em torno da enfermagem como ciência e da necessidade e estratégias de desenvolvimento de teorias de enfermagem. Como exemplo entre as várias meta-teorias, podem ser citados os trabalhos de BATEY ${ }^{2}$, GODNER $^{8}$, CHINN \& JACOBS $^{5}$ e MELEIS ${ }^{13}$.

O segundo nível de teorias é a teoria de grande porte. Essas teorias apresentam maneiras globais de perceber um fenômeno e ofenecem perspectivas para a prática de um modo abrangente ${ }^{21}$. A maioria das teorias de enfermagem, desenvolvidas até agora, são consideradas teorias de grande porte. O termo "conceptual framework" (marco conceitual/teórico) é, muitas vezes, usado como sinônimo de teoria de grande porte na literatura americana die enfermagem. Segundo NEUMAN ${ }^{14}$, marco conceitual é um conjunto de definições e de conceitos interrelacionados. Há, no entanto, marcos conceituais de diferentes niveis e com diferentes objetivos. Um determinado marco conceitual pode ter o objetivo de descrever uma área de conhecimento como, por exemplo a enfermagem; um outro marco conceitual pode ter sido desenvolvido com o objetivo de guiar um estudo específico de pesquisa ou um currículo. Esse tipo de marco conceitual ou teórico pode servir, segundo NEVES \& GONÇALVES ${ }^{15}$, para guiar os passos de um estudo de pesquisa, desde a definição do problema até os resultados. $\mathrm{E}$ importante salientar que, quando se trata de um marco conceitual, desenvolvido especificamente com a finalidade de servir de guia para uma pesquisa ou curriculo, este não é sinônimo de teoria de grande porte. $\mathrm{Na}$ enfermagem, por exemplo, temos vários marcos conceituais que podem ser chamados teorias de grande porte. Entre eles, pode-se citar as teorias de Sister Callista Roy, Betty Newman, Martha Rogers, Dorothea Orem, Wanda Horta e outros. A maioria dos rnnceitos-chave destas teorias de enfermagem são construtos; portanto, são altamente abstratos, de difícil operacionalização. Por isso, dificilmente, pode-se testar empiricamente uma teoria de grande porte como um todo. Entretanto, teorias menos abrangentes do que estas, podem ser derivadas das teorias de grande porte, as quais podem ser testadas na sua totalidade. 
O terceiro nível de teorias é caracterizado pelas teorias de médio porte, que são mais limitadas na sua abrangência do que as teorias de grande porte. Os conceitos desse tipo de teoria são de fácil operacionalização e, por isso, tais teorias podem ser testadas empiricamente ${ }^{3}$. Um exemplo, talvez, esclaneça melhor a diferença entre uma teoria de grande porte (marco conceitual) e uma teoria de médio porte. Uma teoria de grande porte como, por exemplo, a teoria de enfermagem de ROY ${ }^{19}$, descreve e explica o fenômeno da enfermagem, e como conceito de "enfermagem" é amplo, esta teoria pode ser aplicada em qualquer sub-área da enfermagem e para qualquer tipo de assistência de enfermagem. Uma teoria de médio porte, como por exemplo a teoria de "stress" de Selye, é menos abrangente do que a teoria de enfermagem de Roy, isto porque só é aplicada a situaçōes de stress.

O quarto nivel de teorias consiste nas de pequeno porte. São elas menos abrangentes do que as de médio porte. Por exemplo, poderia existir uma teoria que tratasse do stress das pessoas no periodo pré-operatório. Essa teoria poderia descrever o stress experimentado no pré-operatório e propor açōes de enfermagem específicas para prevenir o stress nesta situação. Neste caso, teríamos uma teoria de enfermagem sobre stress. Vejamos a diferença entre teoria de médio e pequeno porte. A teoria do stress de médio porte pode ser aplicada em qualquer situação de stress, enquanto que a teoria de pequeno porte só poderia ser aplicada para situações específicas de stress; nesse caso, no período pré-operatório.

Como já foi mencionado anteriormente, vários marcos conceituais ou teorias de grande porte foram desenvolvidos na enfermagem. No entanto, tem sido notado que o pessoal de enfermagem tem enfrentado certa dificuldade em praticar enfermagem de acordo com estas teorias. Essa dificuldađe pode, talvez, ser atribuída, em parte, à falta de conceituações e definições nos marcos conceituais que possam guiar a prática de enfermagem em todas as suas sub-áreas e/ou em casos mais específicos. Mas, quer isso dizer que as teorias de enfermagem são falhas e/ou devam ser rejeitadas? Para responder a esta pergunta, retornamos à definição de marco conceitual que é um conjunto de definiçōes e conceitos, interrelacionados com o objetivo de apresentar maneiras globais de perceber um fenômeno e de guiar a prática de um modo abrangente, 14,20. Assim os marcos conceituais de enfermagem desenvolvidos até agora, dão uma visão global da enfermagem, isto se justifica porque, afim de desenvolver um corpo de conhecimento de uma profissão, é imprescindível que se inicie com conceitualizações mais abrangentes que sirvam de marcos para a construção e desenvolvimento de um corpo de conhecimento, o qual inclui teorias dos três níveis.

As teorias de grande porte ou marcos conceituais desenvolvidos na enfermagem não são destinados a dar direções em relação a como previnir, solucionar, ou aliviar um problema através de uma maneira própria da enfermagem. Talvez seja este o fato que leve alguns profissionais de enfermagem a não aceitarem as teorias de enfermagem e até a sentirem certa frustração por perceberem uma lacuna entre teoria e prática de enfermagem. 
Será que essa lacuna existente entre a grande teoria e a prática de enfermagem não é devida à falta de teorias de médio e pequeno porte na enfermagem? As teorias de médio e pequeno porte podem ser desenvolvidas a partir das grandes teorias, da pesquisa e/ou da prática. As grandes teorias marcaram um grande avanço para a enflermagem, acredita-se que, quando a enfermagem tiver também teorias de médio e pequeno porte, terá desenvolvido seu próprio corpo de conhecimentos completamente, a autonomia profissional será uma conseqüência do uso de teorias próprias na prática, o que dará à enfermagem condições de predizer eficientemente as conseqüências de assistência ${ }^{13}$.

\section{Relação entre Teoria, Pesquisa e Prática de Enfermagem.}

Pesquisa, segundo ANDER-EGG ${ }^{1}$, é um procedimento reflexivo, sistemático, que permite descobrir novos fato, relações entre variáveis, os quais constituem um caminho para o conhecimento da realidade. A pesquisa pode ser conduzida para testar relações entre conceitos previamente propostos, ou como um método para gerar novos conceitos e estabelecer relações entre esses conceitos, com o fim de construir uma teoria ${ }^{12}$. Pesquisa é também vista como um processo que envolve uma seqüência de passos distintos, a qual é iniciada com a definição do problema de pesquisa e termina com a divulgação dos resultados ${ }^{17}$.

Para CHINN \& JACOBS 5 , a pesquisa pode ou não estar ligada à teoria. A pesquisa que não está ligada à teoria é denominada pesquisa isolada. Esse tipo de pesquisa inclui as de caráter exploratório e os "surveys". A pesquisa ligada à teoria pode ser dividida em três tipos. O primeiro tipo é constituído pelas pesquisas derivadas ou guiadas pela teoria. Neste caso, o problema de pesquisa pode ser derivado diretamente de uma teoria. Por exemplo, alguém poderia querer pesquisar a habilidade das pessoas com câncer para o auto-cuidado, segundo a teoria de Orem. Assim, a teoria da Orem serviria de guia para a pesquisa. Por outro lado, um problema de pesquisa pode não ter sido derivado diretamente de uma teoria, mas pode ser estudado ou visto segundo uma determinada teoria. O segundo tipo de pesquisa é a que tem como objetivo testar uma teoria ou parte de uma teoria. A teoria propõe explicar, predizer e controlar um fenômeno e a pesquisa testa empiricamente essa explicação, predição e controle. $O$ terceiro tipo de pesquisa é a que tem como objetivo construir uma teoria. Esse tipo de pesquisa é visto como um método inđutivo de construir teoria. Há vários métodos para conduzi.' esse tipo de pesquisa. O método mais conhecido na enfermagem é o método apresentado por GLASER \& STRAUSS 7, denominado "Grounded theory", que tem sido usado para desenvolver teorias de médio e pequeno porte.

Vejamos, agora, como a prática está relacionada com a teoria e a pesquisa. As idéias surgem de experiências da vida, como por exemplo, de observações; em outras palavras, as idéias sempre surgem da prática. As teorias e os problemas de pesquisa, portanto, têm suas origens na prática. A teoria dirige e explica a prática. A prática, dirigida pela teoria, redefine a teoria ou a rejeita. A teoria redefinida volta a ser aplicada na prática ${ }^{20}$. Por outro lado, a prática gera problemas para pesquisa; os resul- 
tados da pesquisa, por sua vez, sảo utilizados na prática. Assim, teoria, pesquisa e prática interagem, uma melhorando a outra. Teoria e pesquisa vêm da prática, e são melhoradas na prática 4 . Com a teoria e pesquisa melhoradas, teremos uma prática melhor. Uma boa prática leva a nova pesquisa e a uma nova ou redefinida teoria. No caso das teorias de enfermagem, por exemplo, deveriam ser usadas na prática, pois só a aplicação na prática poderia oferecer base para uma redefinição, rejeição ou confirmação das teorias de enfermagem. As teorias de enfermagem foram desenvolvidas, não para serem impostas, mas para serem testadas na prática. Tendo em vista que a maioria das teorias de enfermagem são teorias de grande porte e, por isso, não podem ser testadas empiricamente como um todo, poderiam ser submetidas parcialmente a testes na prática de enfermagem.

FAWCETT ${ }^{6}$ compara a relação entre teoria e pesquisa a uma hélice dupla. A teoria é vista como uma hélice que gira em espiral, começando com a concepção de uma idéia e sofrendo modificações ou, então, sendo rejeitada. Para FAWCETT ${ }^{6}$, a pesquisa é a segunda hélice, que gira também em espiral, e que começa com a identificação do problema da pesquisa e acompanha o seu processo até os resultados e recomendações.

A relação dinâmica entre teoria, pesquisa e prática é representada, neste trabalho, como uma espiral triplice (figura 1). Vamos denominar essas espirais como: 1) espiral-teoria, 2) espiral-pesquisa e 3) espiral-prática. Cada um dos espirais tem seus próprios objetivos; mas, por outro lado, interagem de maneira dinâmica para um crescimento mútuo. A teoria direciona a pesquisa e a prática, explica a prática, e gera problemas de pesquisa. A pesquisa gera, testa e redefine a teoria. A teoria redefinida volta a ser aplicada na prática, e os resultados da pesquisa são usados para melhorar a prática. Assim, teoria, pesquisa e prática interagem, uma tornando a outra melhor. Teoria e pesquisa "nascem" da prática e são aplicadas na prática.

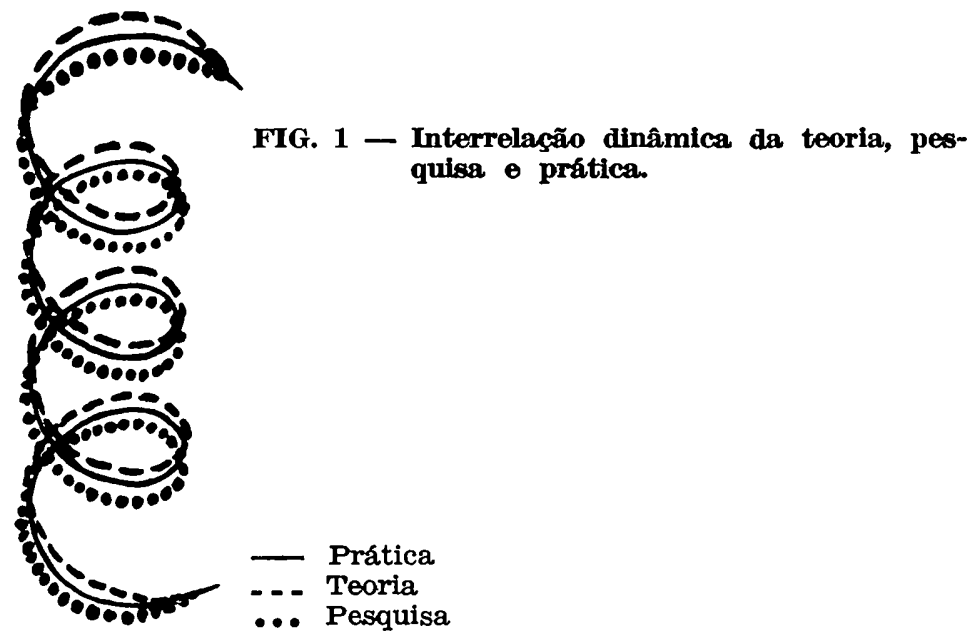


TRENTINI, M. The relationship among theory, research and practice in nursing. Rev. Esc. Enf. USP, São Paulo, $21(2): 135-143$, Aug. 1987.

The present study is an attempt to present a process of relationship among theory, research, and practice in nursing. First, the term theory is defined as well as each of the theory's basic elements. Secondly, the concepts of research and practice are discussed in terms of their potential for stemming, testing, and redefining the theory. Thirdly, the concepts of theory, research and practice are presented as a triple spiral which works as a dinamic process. Within this process the theory, research, and practice interact to improve each other.

UNITERMS: Nursing theory. Nursing research. Nursing practice.

\section{REFERENCIAS BIBLIOGRAFICAS}

1. ANDER-EGG, E. Introducción a las técnicas de investigación social para trabajadores sociales. Buenos Aires, Humanitas, 1978.

2. BATEY, M.V. Conceptualization: knowledge and logic guiding empirical research. Nurs. Res., New York, 26(5):324-9, 1977.

3. BECKSTRAND, J. The need for a practice throry as indicated by the knowledge used in the conduct of practice. Res. Nurs. Health. New York, 4(1):175-9, 1978.

4. BENOLIEL, J.Q. The interaction between theory and research. Nurs. Outlook, New York, 25(2):108-13, 1977.

5. CHINN, P.L. \& JACOBS, M.K. Theory and nursing: a systematic approach. Saint Louis, Mosby, 1983.

6. FAWCET, J. The relationship between theory and research: a double helix. Adv. Nurs. Sci., Germantown, 1(1):49-62, 1978.

7. GLASER, B.g. \& STRAUSS, A.L. The discovery of grounded theory: strategies for qualitative research. New York, Aldine Publishing, 1967.

8. GODNER, S.R. Nursing science in transition. Nurs. Res., New York, 29(3):176-39, 1980.

9. HARDY, M.E. Perspectives on nursing theory. Adv. Nurs. Sci., Germantown, 1(1):37-48, 1978.

10. JACOX, A. Theory contruction in nursing: an overview. Nurs. Ros., New York, 23(1): 4-13, 1974.

11. KERLINGER, F.N. Foundation of behavioral research. New York, Holt, Rinehart and Winston, 1973.

12. MARX, M.H. Formal theory. In: MARX, M.H. \& GODDSON, F. Theory in contemparacy psychology. New York, MacMillan, 1976.

13. MELEIS, A.J. Theoretical nursing: development and progress. Philadelphia, J.B. Lippincott, 1985.

14. NEUMAN, B. The Neuman systems model: application to nursing education and practice. Norwalk, Appleton-Century-Crofts, 1982.

15. NEVES, E.P. \& GONÇALVES, L.H.T. As questões do marco teórico nas pesquisas de enfermagem. In: SEMINARIO DE PESQUISA EM ENFERMAGEM, 3॰, Florianópolis, 1984. Anais. Florianópolis, Ed. da UFSC, 1984.

16. OREM, D.G. Nursing: concepts of practice. New York, McGraw-Hill Book, 1985. 
17. POLIT, D. \& HUNGLER, B. Nursing research: principles and methods. Philadelphia, J.B. Lippincott, 1978.

18. REYNOLDS, P.D. A primer in theory construction. Indianópolis, Bobbs-Merrill Educational Publishing, 1971.

19. ROY, C. \& ROBERTS, S.L. Theory construction in nursing: an adaptation model. Englewoods Cliffs Prentice-Hall, 1981.

20. STEVENS, B.J. Nursing theory: analysis, application, evaluation. Boston, Little Brown, 1979.

21. WAIKEER, L.O. \& AVANT, K.C. Strategies for theory construction in nursing. Norwalk, Appleton-Century-Crofts, 1983.

22. WILLIAMS, C. The nature and development of conceptual frameworks. In: BOWNS, F.S. \& FLEMING, J.W. Issues in nursing research. Norwalk, Appleton-Century-Crofts, 1979.

Entregue para publicação em 10-08-86

1provado para publicação em 17-08-87 